\title{
Banach spaces of universal Taylor series in the disc algebra
}

\author{
Luis Bernal-González, Andreas Jung and Jürgen Müller
}

\begin{abstract}
It is proved that there are large vector spaces of functions in the disc algebra for which every nonzero member satisfies that, for many small subsets $E$ of the unit circle $\mathbb{T}$, the restrictions to $\mathbb{T}$ of the partial sums of its Taylor series at the origin approximate any prescribed function on $E$. Moreover, it is shown that such sets necessarily have to be small in terms of porosity.
\end{abstract}

Mathematics Subject Classification (2010). Primary 47A16; Secondary 15A03, 40A30, 42A16, 46E10.

Keywords. Disc algebra, Taylor series, Fourier series, lineability, spaceability, universal divergence.

\section{Introduction, notation and known results}

Let $\mathbb{T}$ denote the unit circle $\{z \in \mathbb{C}:|z|=1\}=\left\{e^{i t}: t \in[0,2 \pi]\right\}$ in the complex plane $\mathbb{C}$, and let $C(E)$ be the Banach space of continuous functions $E \rightarrow \mathbb{C}$ endowed with the maximum norm $\|f\|_{\infty}=\sup _{E}|f|$, where $E$ is a compact space. The existence of functions $f \in C(\mathbb{T})$ whose Fourier series $\sum_{k=-\infty}^{\infty} \widehat{f}(k) e^{i k t}$ diverge at a point $x_{0}=e^{i t_{0}}$ is known from Du Bois-Reymond (1873) (see e.g. [15, pp. 67-73]). One can even get unbounded divergence, that is, the sequence $\left\{S_{n}\left(f, t_{0}\right):=\sum_{k=-n}^{n} \widehat{f}(k) e^{i k t_{0}}\right\}_{n \geq 1}$ of partial Fourier sums is unbounded. This property is topologically generic: the functions $f$ satisfying it form a residual subset of $C(\mathbb{T})$ (see more in [12]). It is well known that, if $E \subset \mathbb{T}$, then the set $\mathcal{F}_{E}:=\left\{f \in C(\mathbb{T}):\left\{S_{n}(f, t)\right\}_{n \geq 1}\right.$ is unbounded for each $\left.e^{i t} \in E\right\} \neq \emptyset$ if and only if $E$ has Lebesgue measure zero (see [8, 13]).

We will need some terminology from lineability theory (see [1] and references in it). Assume that $A$ is a subset of a vector space $X$. Then $A$ is called lineable if there is an infinite-dimensional vector space $M$ such that

The first author has been partially supported by Plan Andaluz de Investigación de la Junta de Andalucía FQM-127 Grant P08-FQM-03543 and by MEC Grant MTM2015-65242-C21-P. The second author has been supported by the Stipendienstiftung Rheinland-Pfalz. 
$M \backslash\{0\} \subset A$. If, in addition, $X$ is a topological vector space, we say that $A$ is dense-lineable (spaceable, resp.) provided that there exists a dense (a closed infinite-dimensional, resp.) vector subspace $M$ with $M \backslash\{0\} \subset A$. The set $A$ is called maximal dense-lineable if $M \backslash\{0\} \subset A$ for some dense vector subspace $M$ with $\operatorname{dim}(M)=\operatorname{dim}(X)$.

Bayart [2,3] has proved that if $E \subset \mathbb{T}$ has Lebesgue measure zero, the family $\mathcal{F}_{E}$ is dense-lineable and spaceable. In 2010, the third author showed in [16] that, for small subsets $E$ of $\mathbb{T}$, the divergence of the Fourier series of a function $f \in C(\mathbb{T})$ may be "maximal", in the sense that the partial sums are not only unbounded but also present universal properties. In fact, he proved that topological genericity is kept. The corresponding algebraic genericity was obtained by the first author in 2012, see [6]. To be more precise, the main results of [16] and [6] can be stated as in Theorem 1.1 below, but let us first save some notation.

Assume that $E \subset \mathbb{T}$. Let us consider the space $\mathbb{C}^{E}$ of all functions $E \rightarrow \mathbb{C}$ endowed with the topology of the pointwise convergence. Assume that $E$ is countable. Then $\mathbb{C}^{E}$ is a (separable) Fréchet space, i.e. a metrizable complete locally convex topological vector space. We define $\mathcal{U}_{p E}$ as the set of continuous functions whose Fourier sums are pointwise universal on $E$, that is,

$$
\mathcal{U}_{p E}:=\left\{f \in C(\mathbb{T}):\left\{\left.S_{n}(f, \cdot)\right|_{E}\right\}_{n \geq 1} \text { is dense in } \mathbb{C}^{E}\right\} \subset \mathcal{F}_{E} .
$$

Next, let $E \subset \mathbb{T}$ be a compact set and $\mathcal{U}_{u E}$ be the family of continuous functions on $\mathbb{T}$ having Fourier series which are uniformly universal on $C(E)$, that is

$$
\mathcal{U}_{u E}:=\left\{f \in C(\mathbb{T}):\left\{\left.S_{n}(f, \cdot)\right|_{E}\right\}_{n \geq 1} \text { is dense in }\left(C(E),\|\cdot\|_{\infty}\right)\right\} .
$$

Define the set

$$
\begin{gathered}
\mathcal{U K}:=\left\{f \in C(\mathbb{T}): \text { there exists a residual subset } \mathcal{E}_{f} \text { of } \mathcal{K}(\mathbb{T})\right. \\
\text { with } \left.f \in \mathcal{U}_{u E} \text { for all } E \in \mathcal{E}_{f}\right\},
\end{gathered}
$$

where $\mathcal{K}(S)$ denotes the (complete) metric space of all nonempty compact subsets of $S$ endowed with the Hausdorff metric and $S$ is a compact metric space (see [17]). Under this notation, we have the following result, see $[6,16]$.

Theorem 1.1. (a) For each countable set $E \subset \mathbb{T}$ the set $\mathcal{U}_{p E}$ is residual, maximal dense-lineable and spaceable in $C(\mathbb{T})$.

(b) The set $\mathcal{U K}$ is residual, maximal dense-lineable and spaceable in $C(\mathbb{T})$.

Let $\mathbb{D}$ and $\overline{\mathbb{D}}$ denote, respectively, the open unit disc and its closure, so that $\overline{\mathbb{D}}=\mathbb{D} \cup \mathbb{T}$. Consider the disc algebra $A(\mathbb{D})$ consisting of those continuous functions $\overline{\mathbb{D}} \rightarrow \mathbb{C}$ that are holomorphic in the open unit disc $\mathbb{D}$; see e.g. [11, Chap. 6]. Then $A(\mathbb{D})$ becomes a Banach space under the norm $\|f\|=\max \{|f(z)|: z \in \overline{\mathbb{D}}\}$. It is well known that a function $f \in C(\mathbb{T})$ can be extended to a function of $A(\mathbb{D})$ if and only if $\widehat{f}(-k)=0(k \geq 1)$. In this case, the Fourier series of $f$ is the restriction to $\mathbb{T}$ of the Taylor series of $f$ at the origin, so that $\widehat{f}(k)=f^{(k)}(0) / k$ ! for $k \geq 0$. Hence $A(\mathbb{D})$ can be considered 
as a (small) closed subspace of $C(\mathbb{T})$ (recall that, by the maximum modulus principle, $\|f\|=\max _{\mathbb{T}}|f|=\left\|\left.f\right|_{\mathbb{T}}\right\|_{\infty}$ for every $\left.f \in A(\mathbb{D})\right)$. For $E \subset \mathbb{T}$ we set

$$
\begin{aligned}
& A \mathcal{U}_{p E}:=\left\{f \in A(\mathbb{D}):\left\{\left.S_{n}(f, \cdot)\right|_{E}\right\}_{n \geq 1} \text { is dense in } \mathbb{C}^{E}\right\}(E \text { countable }) \\
& A \mathcal{U}_{u E}:=\left\{f \in A(\mathbb{D}):\left\{\left.S_{n}(f, \cdot)\right|_{E}\right\}_{n \geq 1} \text { is dense in } C(E)\right\}(E \text { compact })
\end{aligned}
$$

and moreover we define

$$
\begin{aligned}
A \mathcal{U K}:=\{f \in A(\mathbb{D}): & \text { there exists a residual subset } \mathcal{E}_{f} \text { of } \mathcal{K}(\mathbb{T}) \\
& \text { such that } \left.f \in A \mathcal{U}_{u E} \text { for all } E \in \mathcal{E}_{f}\right\} .
\end{aligned}
$$

Of course, $A \mathcal{U}_{p E} \subset \mathcal{U}_{p E}, A \mathcal{U}_{u E} \subset \mathcal{U}_{u E}$ and $A \mathcal{U} \mathcal{K} \subset \mathcal{U} \mathcal{K}$.

Herzog and Kunstmann [10, Theorem 1] have shown that the topological genericity of $\mathcal{F}_{E}$ given in Theorem 1.1(a) has its counterpart in the disc algebra:

Theorem 1.2. If $E \subset \mathbb{T}$ is countable then the set $A \mathcal{U}_{p E}$ is residual in $A(\mathbb{D})$.

Our first aim is to establish an $A(\mathbb{D})$-analogue of Theorem 1.1 and to complement Theorem 1.2 so as to show that the property established in the latter theorem is generic not only in a topological sense but even in an algebraic sense. In Section 3 we turn to the question which compact sets $E$ have the property that $A \mathcal{U}_{u E}$ is nonempty. In particular, it is shown that a strong form of porosity turns out to be necessary.

\section{Subspaces of maximally divergent $A(\mathbb{D})$-Taylor series on $\mathbb{T}$}

We start with some necessary background. Let $X$ and $Y$ be two Hausdorff topological spaces and $T_{n}: X \rightarrow Y(n \in \mathbb{N}:=\{1,2, \ldots\})$ be a sequence of continuous mappings. Then $\left(T_{n}\right)$ is said to be universal provided that there exists an element $x_{0} \in X$, called universal for $\left(T_{n}\right)$, such that the orbit $\left\{T_{n} x_{0}: n \in \mathbb{N}\right\}$ is dense in $Y$. We set $\mathcal{U}\left(\left(T_{n}\right)\right):=\{x \in X: x$ is universal for $\left.\left(T_{n}\right)\right\}$. Then $\left(T_{n}\right)$ is said to be densely universal if $\mathcal{U}\left(\left(T_{n}\right)\right)$ is dense in $X$, and hereditarily densely universal if $\left(T_{n_{k}}\right)$ is densely universal for every strictly increasing sequence $\left(n_{k}\right) \subset \mathbb{N}$.

Theorem 2.1. ([9, Chap. 10]) Let $X, Y$ be separable Fréchet spaces such that $X$ supports a continuous norm, and let $T_{n}: X \rightarrow Y$ be continuous linear mappings. Suppose that the sequence $\left(T_{n}\right)$ is hereditarily densely universal and that there exists a closed infinite-dimensional vector subspace $M$ of $X$ such that the sequence $\left(T_{n} x\right)_{n \geq 1}$ converges in $Y$ for every $x \in M$. Then the set $\mathcal{U}\left(\left(T_{n}\right)\right)$ is spaceable.

Remark 2.2. We fix a (countable) set $E=\left\{e^{i t_{j}}: j \in \mathbb{N}\right\} \subset \mathbb{T}$ and consider the sequence of linear mappings $T_{n}:\left.f \in A(\mathbb{D}) \mapsto S_{n}(f, \cdot)\right|_{E} \in \mathbb{C}^{E}(n \geq 1)$. They are continuous because $\left|T_{n} f(\xi)\right| \leq(n+1)\|f\|_{\infty}(\xi \in E)$, this inequality being true due to Cauchy's inequalities $\left|f^{(j)}(0) / j !\right| \leq\|f\|_{\infty}(j \geq 0)$. We make use of Theorem 1.2, but in a stronger form. The proof in [10] depends ultimately on the fact that $S_{n}\left(R_{n}, 1\right) \longrightarrow \infty$ as $n \rightarrow \infty$, where the $R_{n}$ 's are 
the so-called Landau extremal functions. This implies that the sequence $\left(T_{n}\right)$ is hereditarily densely universal.

The following criterion for discovering dense-lineability from mere lineability can be found in [1, Chap. 7].

Theorem 2.3. Assume that $X$ is a metrizable separable topological vector space. Let $\alpha$ be an infinite cardinal number. Suppose that $A, B \subset X$ are two subsets such that $A \cup\{0\}$ contains an $\alpha$-dimensional vector space, $B$ is a dense vector subspace, $A+B \subset A$ and $A \cap B=\emptyset$. Then $A \cup\{0\}$ contains a dense vector space $D$ with $\operatorname{dim}(D)=\alpha$.

We are now ready to state the main result of this section.

Theorem 2.4. (a) For each countable set $E \subset \mathbb{T}$ the set $A \mathcal{U}_{p E}$ is maximal dense-lineable and spaceable in $A(\mathbb{D})$.

(b) $A \mathcal{U K}$ is residual, maximal dense-lineable and spaceable in $A(\mathbb{D})$.

Proof. (a) We first note that $A \mathcal{U}_{p E}=\mathcal{U}\left(\left(T_{n}\right)\right)$. Now, let $M$ be the closed linear span in $C(\mathbb{T})$ of the functions $e^{i t} \mapsto e^{i 2^{k} t}(k \geq 1)$. Then $M$ is a closed infinite-dimensional vector subspace of $C(\mathbb{T})$ such that $S_{n}(f, t) \underset{n \rightarrow \infty}{\longrightarrow} f\left(e^{i t}\right)$ uniformly on $[0,2 \pi]$ for every $f \in M$ (see [6, Lemma 3.1]). Since the functions $z \mapsto z^{2^{k}}(k \geq 1)$ are in $A(\mathbb{D})$ and $A(\mathbb{D})$ carries the topology inherited from $C(\mathbb{T})$, we get a closed infinite-dimensional vector subspace $\widetilde{M} \subset A(\mathbb{D})$ such that $\left(\left.S_{n} f\right|_{\mathbb{T}}\right)$ converges uniformly to $\left.f\right|_{\mathbb{T}}$ for all $f \in \widetilde{M}$. In particular, the sequence $\left\{\left.S_{n}(f, \cdot)\right|_{E}\right\}_{n \geq 1}$ converges in $\mathbb{C}^{E}$ for all $f \in \widetilde{M}$. Then the spaceability of $A \mathcal{U}_{p E}$ is a direct consequence of the fact that $A \mathcal{U}_{p E}=\mathcal{U}\left(\left(T_{n}\right)\right)$ and Theorem 2.1 (with $X=A(\mathbb{D})$ and $Y=\mathbb{C}^{E}$ ).

Next, we are going to prove the maximal dense-lineability of $A \mathcal{U}_{p E}$. To this end, note that from the spaceability of the same set we can fix a closed infinite-dimensional vector subspace $L$ of $A(\mathbb{D})$ with $L \subset A \mathcal{U}_{p E} \cup\{0\}$. Since $L$ is a separable F-space, an application of Baire's category theorem yields $\operatorname{dim}(L)=\mathfrak{c}=\operatorname{dim}(A(\mathbb{D}))$, where $\mathfrak{c}$ denotes cardinality of the continuum. Thus, it is enough to apply Theorem 2.3 with $\alpha=\mathfrak{c}, X=A(\mathbb{D}), A=A \mathcal{U}_{p E}$, $B=\{$ the polynomials in $z\}$. Indeed, on the one hand, it is plain that no polynomial has a universal sequence of Taylor partial sums, so $A \cap B=\emptyset$. On the other hand, if $P$ is a polynomial and $f \in A \mathcal{U}_{p E}$ then, given $\varphi \in$ $\mathbb{C}^{E}$, there is $\left(n_{k}\right) \subset \mathbb{N}$ with $S_{n_{k}}(f, \cdot) \longrightarrow \varphi-P(k \rightarrow \infty)$ pointwise on $E$. But $S_{n}(P, \cdot)=P$ for all $n \geq \operatorname{degree}(P)$. Therefore, we get for $k$ large that $S_{n_{k}}(f+P, \cdot)=P+S_{n_{k}}(f, \cdot)$, which tends to $\varphi$ as $k \rightarrow \infty$ in $\mathbb{C}^{E}$. Thus, $f+P \in A \mathcal{U}_{p E}$ or, under our terminology, $A+B \subset A$, as required.

(b) We follow the approach of [16, proof of Lemma 2]. Fix a dense countable set $D \subset \mathbb{T}$ and denote by $\mathcal{E}$ the family of all finite subsets of $D$. Then $\mathcal{E}$ is countable and dense in $\mathcal{K}(\mathbb{T})$. By Theorem $1.2, A \mathcal{U}_{p D}$ is a residual subset of $A(\mathbb{D})$. Now, for each $f \in C(\mathbb{T})$ (and, in particular, for each $f \in A(\mathbb{D})), \mathcal{E}_{f}:=\left\{E \in \mathcal{K}(\mathbb{T}):\left\{\left.S_{n}(f, \cdot)\right|_{E}\right\}_{n \geq 1}\right.$ is dense in $\left.C(E)\right\}$ is a $G_{\delta}$ subset of $\mathcal{K}(\mathbb{T})$ (see [16]). But $\mathcal{E} \subset \mathcal{E}_{f}$ for all $f \in \mathcal{U}_{p D}$, hence for all $f \in A \mathcal{U}_{p D}$, 
so $\mathcal{E}_{f}$ is residual in this case. Since $A \mathcal{U K}=\left\{f \in A(\mathbb{D}): \mathcal{E}_{f}\right.$ is residual $\}$, one gets $A \mathcal{U}_{p D} \subset A \mathcal{U} \mathcal{K}$. An application of Theorem 1.2 and of part (a) of the current theorem to $E=D$ concludes the proof.

We can take advantage of the fact that for countable $E \subset \mathbb{T}$ the sequence $\left(T_{n}\right)$ is hereditarily densely universal to prove a result about an interesting operator, namely, the backward shift

$$
B: f(z)=\sum_{k=0}^{\infty} a_{k} z^{k} \in A(\mathbb{D}) \mapsto \sum_{k=0}^{\infty} a_{k+1} z^{k} \in A(\mathbb{D}) .
$$

It is easy to see that $B$ defines a (linear, continuous) operator on $A(\mathbb{D})$. The hypercyclicity of the operator $B: X \rightarrow X$ has been extensively studied in many spaces $X$, see e.g. [9] (recall that an operator $T: X \rightarrow X$ is called hypercyclic whenever the sequence $\left(T^{n}\right)$ of its iterates is universal). For instance, for the Hardy space

$$
H^{2}=\left\{f(z)=\sum_{k=0}^{\infty} a_{k} z^{k} \in H(\mathbb{D}):\|f\|_{2}:=\left(\sum_{k=0}^{\infty}\left|a_{k}\right|^{2}\right)^{1 / 2}<+\infty\right\},
$$

the operator $B$ is not hypercyclic because $\|B\|=1$. An easy argument using the fact that $A(\mathbb{D})$ is continuously and densely embedded in $H^{2}$ implies that $B$ is not hypercyclic on $A(\mathbb{D})$ either. Nevertheless, we may preserve universality on small subsets of $\mathbb{T}$.

Theorem 2.5. Let $E \subset \mathbb{T}$ be a countable set and let $\left(n_{k}\right) \subset \mathbb{N}$ be a strictly increasing sequence. Then there is a residual subset of functions $f$ in $A(\mathbb{D})$ such that the set $\left\{\left.\left(B^{n_{k}} f\right)\right|_{E}: k \geq 1\right\}$ is dense in $\mathbb{C}^{E}$.

Proof. Let $E=\left\{\xi_{1}, \xi_{2}, \xi_{3}, \ldots\right\}$. Fix $\xi \in \mathbb{T}$. Then, for a given subsequence $\left(n_{k}\right) \subset \mathbb{N}$, the sequence $\left\{\xi^{n_{k}}: k \geq 1\right\}(\subset \mathbb{T})$ is bounded. Therefore it contains a convergent subsequence. Applying successively this result to $\xi=\xi_{1}, \xi_{2}, \ldots$, a diagonalization process produces a subsequence $\left(m_{k}\right)$ of $\left(n_{k}\right)$ such that, for each $\xi \in E$, the sequence $\left\{\xi^{m_{k}}\right\}_{k \geq 1}$ converges (to $\varphi(\xi)$, say). Since $\left(T_{n}\right)$ is hereditarily densely universal, there is a dense - and, according to the Universality Criterion (see [9]), residual - set $\mathcal{R} \subset A(\mathbb{D})$ satisfying that $\left\{\left.S_{m_{k}-1}(f, \cdot)\right|_{E}: k \geq 1\right\}(f \in \mathcal{R})$ is dense in $\mathbb{C}^{E}$. Take $f \in \mathcal{R}$ and fix $g \in \mathbb{C}^{E}$. Define $h \in \mathbb{C}^{E}$ as $h:=f-\varphi g$. Then there is a subsequence $\left(p_{k}\right)$ of $\left(m_{k}\right)$ such that $S_{p_{k}-1}(f, \xi) \longrightarrow h(\xi)(k \rightarrow \infty)$ for all $\xi \in E$. Finally, the identity

$$
\left(B^{n} f\right)(\xi)=\frac{f(\xi)-S_{n-1}(f, \xi)}{\xi^{n}}(\xi \in E, n \in \mathbb{N})
$$

together with the definition of $\varphi$ and $h$ shows that $\left(B^{p_{k}} f\right)(\xi) \longrightarrow g(\xi)(k \rightarrow$ $\infty)$ pointwise on $E$. This proves the theorem.

Remark 2.6. If we consider the backward shift $B$ as a mapping defined on the Hardy space $H^{2}$ instead of $A(\mathbb{D})$, we obtain universality on much larger sets $E$. According to Carleson's theorem we have $S_{n}(f, \cdot) \rightarrow f$ almost everywhere on $\mathbb{T}$ for all $f \in H^{2}$, so we can again expect universality only on sets of vanishing measure. On the other hand, Theorem 1.1 in [5] shows that the 
sequence $T_{n}: H^{2} \rightarrow C(E)$ is hereditarily densely universal for all compact $E$ of vanishing measure. If now $E$ is a Dirichlet set, that is, for some subsequence $\left(m_{k}\right)$ of the positive integers $\left(z^{m_{k}}\right)$ tends to 1 uniformly on $E$, then the proof of Theorem 2.5 shows that there is a residual subset of functions in $H^{2}$ such that $\left\{\left.\left(B^{m_{k}} f\right)\right|_{E}: k \geq 1\right\}$ is dense in $\left(C(E),\|\cdot\|_{\infty}\right)$. Results on Dirichlet sets and related small subsets of $\mathbb{T}$ can be found e.g. in [7, Section 8.6]. While Dirichlet sets always have vanishing measure, they may be large in the sense that the Hausdorff dimension can be 1; see e.g. [12].

\section{Rogosinski summability and uniform universality}

We now turn to the question for which compact sets $E \subset \mathbb{T}$ the set $A \mathcal{U}_{u E}$ is nonempty. Again, we consider the sequence $\left(T_{n}\right)$ with $T_{n} f:=\left.S_{n}(f, \cdot)\right|_{E}$, but now as a mapping from $A(\mathbb{D})$ to $\left(C(E),\|\cdot\|_{\infty}\right)$. Since $\max _{E}\left|T_{n} f\right| \leq$ $(n+1)\|f\|_{\infty}$ for all $f \in A(\mathbb{D})$, the $T_{n}$ are continuous.

It was noticed in [16] that from the residuality part of Theorem 1.1(b) one derives the existence of uncountable compact subsets $E$ of $\mathbb{T}$ (necessarily having vanishing measure) so that $\left\{\left.S_{n}(f, \cdot)\right|_{E}: n \geq 1\right\}$ is dense in $C(E)$ for some $f \in C(\mathbb{T})$, that is, $\mathcal{U}_{u E}$ is nonempty. The same holds for the case of the disc algebra $A(\mathbb{D})$ (cf. [10]). We will show in Theorem 3.2 below that this "intertwining of roles" between $A(\mathbb{D})$ and $\mathcal{K}(\mathbb{T})$ holds in a strong way. In fact, there are many such fortunate sets $E$. With this aim, we define

$$
A \mathcal{K U}:=\left\{E \in \mathcal{K}(\mathbb{T}): A \mathcal{U}_{u E} \text { is residual in } A(\mathbb{D})\right\} .
$$

We need the following auxiliary result, that is similar to the Ulam-Kuratowski theorem (see e.g. [18, Chap. 15]) relating the property of "being large" with respect to two spaces.

Lemma 3.1. Let $X$ and $Y$ be Baire topological spaces and let $Y$ be secondcountable. Moreover, assume that $S \subset X \times Y$ is a subset such that the sets $\left\{x \in X: S(x, \cdot)\right.$ is a $G_{\delta}$-set in $\left.Y\right\}$ and $\{y \in Y: S(\cdot, y)$ is residual in $X\}$ are residual in $X$ and $Y$, respectively, where we have set $S(x, \cdot)=\{y \in Y$ : $(x, y) \in S\}$ and $S(\cdot, y)=\{x \in X:(x, y) \in S\}$. Then the set $\{x \in X: S(x, \cdot)$ is residual in $Y\}$ is residual in $X$.

Proof. According to the assumption, there exists a residual subset $R$ of $Y$ such that $S(\cdot, y)$ is residual in $X$ for all $y \in R$. As $Y$ is a Baire space, $R$ is dense in $Y$. The second-countability of $Y$ implies that $R$ is also secondcountable and hence, in particular, separable. Thus, there exists a countable subset $Q$ of $R$ which is dense in $R$. Then $Q$ is also dense in $Y$ and the set $X_{0}:=\bigcap_{y \in Q} S(\cdot, y)$ is residual in $X$. For $x_{0} \in X_{0}$, we have $\left(x_{0}, y\right) \in S$ and hence $y \in S\left(x_{0}, \cdot\right)$ for all $y \in Q$. Thus, we obtain $S\left(x_{0}, \cdot\right) \supset Q$ so that the denseness of $Q$ in $Y$ implies that $S\left(x_{0}, \cdot\right)$ is dense in $Y$. The assertion now follows from the assumption that there exists a residual set $X_{1} \subset X$ such that $S(x, \cdot)$ is also a $G_{\delta^{-}}$set in $Y$ for each $x \in X_{1}$.

Theorem 3.2. The set $A \mathcal{K U}$ is residual in $\mathcal{K}(\mathbb{T})$. 
Proof. We apply Lemma 3.1 with $X=\mathcal{K}(\mathbb{T}), Y=A(\mathbb{D})$ and

$$
S=\left\{(E, f) \in \mathcal{K}(\mathbb{T}) \times A(\mathbb{D}):\left\{S_{n}(f, \cdot)\right\}_{n \geq 1} \text { is dense in } C(E)\right\} .
$$

Since $X$ and $Y$ are complete metric spaces, they are both Baire spaces. Moreover, $A(\mathbb{D})$ is separable, so second-countable. Let us denote by $\mathcal{P}$ the set of all complex valued polynomials in two real variables with Gaussian rational coefficients. The complex Stone-Weierstrass theorem asserts that this set is (countable and) dense in $C(E)$ for every $E \in \mathcal{K}(\mathbb{T})$. According to the notation in Lemma 3.1, we have

$$
\begin{aligned}
S(E, \cdot) & =\left\{f \in A(\mathbb{D}):\left\{S_{n}(f, \cdot)\right\}_{n \geq 1} \text { is dense in } C(E)\right\} \\
& =\bigcap_{j \in \mathbb{N}, P \in \mathcal{P}} \bigcup_{n \in \mathbb{N}}\left\{f \in A(\mathbb{D}): \max _{\xi \in E}\left|S_{n}(f, \xi)-P(\xi)\right|<1 / j\right\} \\
& =\bigcap_{j \in \mathbb{N}, P \in \mathcal{P}} \bigcup_{n \in \mathbb{N}} T_{n}^{-1}\left(B_{E}(P, 1 / j)\right),
\end{aligned}
$$

where $B_{E}(g, \varepsilon)$ denotes the open $\|\cdot\|_{\infty}$-ball in $C(E)$ with center $g$ and radius $\varepsilon$. Since $T_{n}^{-1}\left(B_{E}(P, 1 / j)\right)$ is open in $A(\mathbb{D})$ for each triple $(j, P, n)$, the set $S(E, \cdot)$ is a $G_{\delta}$ subset of $A(\mathbb{D})=Y$ for each member $E$ of the (trivially) residual subset $\mathcal{K}(\mathbb{T})$ of $\mathcal{K}(\mathbb{T})=X$. If now $f \in A \mathcal{U} \mathcal{K}$ then, by definition, the set $S(\cdot, f)=\left\{E \in \mathcal{K}(\mathbb{T}):\left\{S_{n}(f, \cdot)\right\}_{n \geq 1}\right.$ is dense in $\left.C(E)\right\}$ is residual in $\mathcal{K}(\mathbb{T})=X$. By Theorem 2.4, the set $A \mathcal{U} \mathcal{K}$ is residual in $A(\mathbb{D})=Y$, and this proves the theorem.

A main ingredient in the proof of Theorem 2.4 is the hereditary universality of the sequence $T_{n}: A(\mathbb{D}) \rightarrow \mathbb{C}^{E}$ for countable sets $E$ (Remark 2.2). In particular, for finite sets $E$ the sequence $T_{n}: A(\mathbb{D}) \rightarrow C(E)$ is hereditarily densely universal. We prove that the finiteness of $E$ actually turns out to be necessary. The main tool is the following extension of a classical summability result due to Rogosinski. The proof runs along the same lines as the proof of the classical result of Rogosinski corresponding to the case of $n_{j}$ being equal to $j$ (see e.g. [14, 21]), so it will be omitted. For an arbitrary (formal) power series $\sum_{k=0}^{\infty} a_{k} z^{k}$ we denote by $S_{n}(z)$ the $n$-th partial sum at the point $z \in \mathbb{C}$.

Lemma 3.3. Let $\left(n_{j}\right)$ be a sequence in $\mathbb{N}$ tending to $\infty$ and suppose $\left(z_{j}\right)$ to be a sequence of complex numbers so that $n_{j}\left(z_{j}-1\right)$ is bounded. If $\sum_{k=0}^{\infty} a_{k} z^{k}$ is a power series with $\left(S_{n}(1)\right)$ being $(C, 1)$-summable to the complex number $s$, then

$$
S_{n_{j}}\left(z_{j}\right)-s-\left(S_{n_{j}}(1)-s\right) z_{j}^{n_{j}} \longrightarrow 0 \quad(j \rightarrow \infty) .
$$

Proposition 3.4. Let $E \subset \mathbb{T}$ with $1 \in E$ and suppose that $\left(S_{n}(1)\right)$ is $(C, 1)$ summable to s. Moreover, let $\left(n_{j}\right)$ be a sequence in $\mathbb{N}$ tending to $\infty$ and $\left(e^{i \alpha_{j}}\right)$ a sequence in $E$ with $\operatorname{dist}\left(\left\{n_{j} \alpha_{j}\right\}, 2 \pi \mathbb{Z}\right)>0$ and $\sup _{j}\left|n_{j} \alpha_{j}\right|<\infty$. If a subsequence of $\left(S_{n_{j}}\right)$ tends to $h$ uniformly on $E$ then $h(1)=s$.

Proof. Note first that $\alpha_{j} \rightarrow 0$ as $j \rightarrow \infty$ and that $\left(n_{j}\left(e^{i \alpha_{j}}-1\right)\right) \sim\left(i n_{j} \alpha_{j}\right)$ is bounded. From the uniform convergence of $\left(S_{n_{j_{m}}}\right)$ to $h$ on $E$ we obtain 
that $S_{n_{j_{m}}}\left(e^{i \alpha_{j_{m}}}\right)$ converges to $h(1)$ as $m \rightarrow \infty$. Passing to an appropriate subsequence of $\left(n_{j_{m}}\right)$ we may assume that $n_{j_{m}} \alpha_{j_{m}} \rightarrow \alpha$ as $m \rightarrow \infty$. Then

$$
S_{n_{j_{m}}}\left(e^{i \alpha_{j_{m}}}\right)-s-\left(S_{n_{j_{m}}}(1)-s\right) e^{i n_{j_{m}} \alpha_{j_{m}}} \rightarrow h(1)-s-(h(1)-s) e^{i \alpha}
$$

and from Lemma 3.3 we have $(h(1)-s)\left(1-e^{i \alpha}\right)=0$. Now, since $e^{i \alpha} \neq 1$ the assertion follows.

Corollary 3.5. Let $E$ be a compact subset of $\mathbb{T}$ so that the sequence $T_{n}$ : $A(\mathbb{D}) \rightarrow C(E)$ is hereditarily universal. Then $E$ is finite.

Proof. Let $E \subset \mathbb{T}$ be compact and infinite. Without loss of generality we may suppose that 1 is an accumulation point of $E$. Let $e^{i \alpha_{j}} \in E, e^{i \alpha_{j}} \rightarrow 1$, $\alpha_{j} \neq 0, \alpha_{j} \rightarrow 0$ with $\left(\left|\alpha_{j}\right|\right)$ strictly increasing. Choose positive integers $n_{j}$ such that $\pi /\left|\alpha_{j}\right| \leq n_{j}<1+\pi /\left|\alpha_{j}\right|$, where we may assume that $\left(n_{j}\right)$ is strictly increasing and $\left|\alpha_{j}\right|<\pi$ for all $j$. Then $n_{j}\left|\alpha_{j}\right| \rightarrow \pi$ as $j \rightarrow \infty$. According to Fejér's theorem, $S_{n}(f, 1)$ is $(C, 1)$-summable to $f(1)$ for all $f \in A(\mathbb{D})$. Hence, Proposition 3.4 shows that $\left(T_{n_{j}}\right)$ is not universal.

Remark 3.6. Suppose $\left(\beta_{k}\right)_{k \in \mathbb{N}}$ to be a strictly increasing sequence of positive numbers tending to infinity. If $\left(\beta_{k+1} / \beta_{k}\right)$ is bounded we say that $\left(\beta_{k}\right)$ has bounded quotients.

Let $\left(\beta_{k}\right)$ have bounded quotients. Then for each $n \in \mathbb{N}$ there is a unique $k_{n} \in \mathbb{N}_{0}$ with

$$
\beta_{k_{n}} / 2<n \leq \beta_{k_{n}+1} / 2
$$

(where $\beta_{0}:=0$ ). If we define $\alpha_{n}:=2 \pi / \beta_{k_{n}+1}$ and if $\beta_{k+1} / \beta_{k} \leq M$ we have $\pi / M \leq \pi \beta_{k_{n}} / \beta_{k_{n}+1}<n \alpha_{n} \leq \pi$ for all $n \in \mathbb{N}$ and thus, in particular, $\operatorname{dist}\left(0,\left\{n \alpha_{n}\right\}\right)>0$. We consider a compact subset $E$ of $\mathbb{T}$ with $1 \in E$ and $E \supset\left\{e^{2 \pi i / \beta_{k}}: k \in \mathbb{N}\right\}$. If $\left(S_{n}(1)\right)$ is $(C, 1)$-summable to $s$ and a subsequence of $\left(S_{n}\right)$ tends to $h$ uniformly on $E$ then Proposition 3.4 (with $n_{j}=j$ ) implies $h(1)=s$. Moreover, $E$ is not a Dirichlet set $^{1}$, since

$$
\left|e^{2 \pi i n / \beta_{k_{n+1}}}-1\right| \geq\left|e^{\pi i / M}-1\right|
$$

and therefore

$$
\max _{z \in E}\left|z^{n}-1\right| \geq\left|e^{\pi i / M}-1\right| .
$$

The same statements hold in the case $E \supset\left\{e^{-2 \pi i / \beta_{k}}: k \in \mathbb{N}\right\}$.

Example. For each $q>1$ the geometric sequence $\left(\beta_{k}\right)=\left(q^{k}\right)$ has bounded quotients. If $E$ is a subset of $\mathbb{T}$ with $1 \in E$ and $E \supset\left\{e^{2 \pi i / q^{k}}: k \in \mathbb{N}\right\}$ then, according to Remark 3.6, the set $E$ is not Dirichlet and for all $\left(S_{n}\right)$ with the property that $\left(S_{n}(1)\right)$ is $(C, 1)$-summable to $s$ we necessarily have $h(1)=s$ for all uniform limits $h$ of subsequences of $\left(S_{n}\right)$ on $E$. For similar considerations we refer also to [19, Section 4].

\footnotetext{
${ }^{1}$ The autors thank the referee for pointing out the direct proof.
} 
We shall give a characterization in terms of porosity of the property that a compact set $E \subset \mathbb{T}$ does not contain a sequence $\left(e^{2 \pi i / \beta_{k}}\right)_{k}$ with $\left(\beta_{k}\right)$ having bounded quotients. For $A \subset \mathbb{R}$ and $x \in \mathbb{R}$ we put

$$
p^{+}(A, x):=\limsup _{r \rightarrow 0} r^{-1} \lambda^{+}(A, x, r),
$$

where $\lambda^{+}(A, x, r)$ denotes the supremum of lengths of open intervals lying in $(x, x+r) \backslash A$. The set $A$ is called porous from the right at the point $x$ if $p^{+}(A, x)>0$. If $p^{+}(A, x)=1$ then $A$ is called strongly porous from the right at $x$ (see, e.g. [20, Chapter 8$]$ ). Similarly, porosity and strong porosity from the left can be defined in terms of $p^{-}(A, x)$, with the interval $(x, x+r)$ replaced by $(x-r, x)$.

Lemma 3.7. Let $E=e^{i A}$ with $0 \in A \subset \mathbb{R}$ compact and $\operatorname{diam}(A)<2 \pi$. Then $A$ is strongly porous at 0 from the right if and only if $E$ does not contain a sequence $\left(e^{2 \pi i / \beta_{k}}\right)_{k}$ with $\left(\beta_{k}\right)$ having bounded quotients. The same statement holds for strong porosity from the left at 0 with $\left(e^{2 \pi i / \beta_{k}}\right)_{k}$ replaced by $\left(e^{-2 \pi i / \beta_{k}}\right)_{k}$.

Proof. 1. Suppose that $E$ does not contain a sequence $\left(e^{2 \pi i / \beta_{k}}\right)_{k}$ with $\left(\beta_{k}\right)$ having bounded quotients. We write $A \cap(0, \infty)=2 \pi / B$ with $B \subset(1, \infty)$ and require without loss of generality that $B$ is unbounded.

For $c>1$ fixed we define a sequence $\left(\beta_{k}\right)$ in $B$ (depending on $c$ ) by recursion. Starting with an arbitrary $\beta_{1} \in B$ and supposing $\beta_{1}, \ldots, \beta_{k}$ to be already defined, we consider the set

$$
B_{k}:=\left\{t \in B: \beta_{k}+1 \leq t<c\left(\beta_{k}+1\right)\right\} .
$$

If $B_{k} \neq \emptyset$, we choose $\beta_{k+1} \in B_{k}$. If $B_{k}=\emptyset$, we choose

$$
\beta_{k+1}:=\min \left\{t \in B: t \geq c\left(\beta_{k}+1\right)\right\} .
$$

Then $\beta_{k+1} \geq \beta_{k}+1$ and thus, in particular, the sequence $\left(\beta_{k}\right)$ increases monotonically to $\infty$. Moreover, the second case appears infinitely often, because otherwise we would have $\beta_{k+1} / \beta_{k}<c+1$ for all $k$ sufficiently large, which would contradict our assumption. This shows that for a subsequence $\left(\beta_{k}\right)_{k \in I}$ (where $\left.I=I(c)\right)$, we have

$$
\frac{\beta_{k+1}}{\beta_{k}} \geq c \frac{\beta_{k}+1}{\beta_{k}}>c
$$

and $\left(\beta_{k}+1, \beta_{k+1}\right) \cap B=\emptyset$ for all $k \in I$.

Taking $c=j+2$ for $j \in \mathbb{N}_{0}$, we can choose $k_{j} \in I(j+2)$ in such a way that for $\gamma_{2 j}:=\beta_{k_{j}}$ and $\gamma_{2 j+1}:=\beta_{k_{j}+1}$ we have $\gamma_{2 j+2}>\gamma_{2 j+1}, \gamma_{2 j+1} / \gamma_{2 j}>j+2$ and $\left(\gamma_{2 j}+1, \gamma_{2 j+1}\right) \cap B=\emptyset$ for $j \in \mathbb{N}_{0}$. From this it follows that $p^{+}(A, 0)=1$.

2. If $A$ is strongly porous at 0 from the right, it follows easily from the definition that $E$ does not contain a sequence $\left(e^{2 \pi i / \beta_{k}}\right)_{k}$ with $\left(\beta_{k}\right)$ having bounded quotients.

3. The case of porosity from the left can be reduced to the former case by considering $-A$ instead of $A$. 
If $E$ is a Dirichlet set and if $\zeta \in E$, then from the Definition it is easily seen that also $\zeta^{-1} E$ is a Dirichlet set. Combining Remark 3.6 and Lemma 3.7 (with $E$ replaced by $e^{-i x} E$ ) we obtain

Theorem 3.8. Let $E=e^{i A}$ with $A \subset(-\pi, \pi)$ compact and suppose that $A$ is not strongly porous at $x \in A$ from the left or the right. If $\left(S_{n}\left(e^{i x}\right)\right)$ is $(C, 1)$ summable to $s$ and if a subsequence of $\left(S_{n}\right)$ tends to $h$ uniformly on $E$ then $h\left(e^{i x}\right)=s$. Moreover, $E$ is not a Dirichlet set.

According to Fejér's theorem, for $f$ in the disc algebra the partial sums $S_{n}(f, \zeta)$ are $(C, 1)$-summable at all $\zeta \in \mathbb{T}$, so the summability condition from Theorem 3.8 is satisfied for arbitrary $x \in A$.

Remark 3.9. If $C$ is the classical Cantor set, it is easily seen that $\pi C$ is not strongly porous from at least one side at all points of the set. So, for $E=e^{\pi i C}$ and for $f$ in the disc algebra the only possible uniform limit function of a subsequence of $\left(S_{n}(f, \cdot)\right)$ is the function $\left.f\right|_{E}$ and thus, in particular, $A \mathcal{U}_{u E}=\emptyset$. In contrast, as already mentioned in Remark 2.6, Theorem 1.1 in [5] shows that the sequence $T_{n}: H^{2} \rightarrow C(E)$ is hereditarily densely universal for $E$.

Corollary 3.10. Let $E=e^{i A}$ with $A \subset(-\pi, \pi)$ compact. Then the set $A$ is strongly porous from the right and the left at all points $x \in A$ if one of the following conditions is satisfied:

1. $A \mathcal{U}_{u E}$ is nonempty.

2. $E$ is a Dirichlet set.

Remark 3.11. In contrast, if $\left(\beta_{k}\right)$ is a sequence with $\beta_{1} \in \mathbb{N}$ and so that $\beta_{k+1}=N_{k} \beta_{k}$ for some unbounded sequence $\left(N_{k}\right)$ of positive integers, then $A=\left\{2 \pi / \beta_{k}: k \in \mathbb{N}\right\}$ is strongly porous at 0 from the right (and of course from the left). Actually, the set $E=\left\{e^{2 \pi i / \beta_{k}}: k \in \mathbb{N}\right\}$ is a Dirichlet set.

(Indeed: Let $I \subset \mathbb{N}$ be so that the subsequence $\left(N_{k}\right)_{k \in I}$ of $\left(N_{k}\right)$ tends to $\infty$. For $m \in I$ we have $e^{2 \pi i \beta_{m} / \beta_{k}}=1$ for all $k \leq m$ and

$$
\sup _{k \geq m+1}\left|e^{2 \pi i \beta_{m} / \beta_{k}}-1\right|=\left|e^{2 \pi i / N_{m}}-1\right| \rightarrow 0 \quad(m \rightarrow \infty, m \in I)
$$

and thus $z^{\beta_{m}} \rightarrow 1$ uniformly on $E$ as $m \rightarrow \infty, m \in I$.)

From Theorem 1.7 and Remark 1.8 in [5] it follows that for each Dirichlet set $E$ there exist power series having the property that $\left(S_{n}(\zeta)\right)$ is $(C, 1)$ summable at all points $\zeta \in E$ (actually on an arc containing $E$ ) and so that $\left\{\left.S_{n}\right|_{E}: n \in \mathbb{N}_{0}\right\}$ is dense in $C(E)$. Up to now, no concrete infinite set $E$ with $A \mathcal{U}_{u E} \neq \emptyset$ is known. The above results suggest that Dirichlet sets might be reasonable candidates.

\section{Acknowledgment}

The authors are grateful to the referee for many helpful comments and suggestions, which improved the presentation considerably. 


\section{References}

[1] R.M. Aron, L. Bernal-González, L., D. Pellegrino and J.B. Seoane-Sepúlveda, Lineability: The search for linearity in Mathematics, Monographs and Research Notes in Mathematics, Chapman \& Hall/CRC, Boca Raton, FL, 2016.

[2] F. Bayart, Topological and algebraic genericity of divergence and universality. Studia Math. 167 (2005), 161-181.

[3] F. Bayart, Linearity of sets of strange functions. Michigan Math. 53 (2005), 291-303.

[4] F. Bayart and E. Matheron, Dynamics of Linear Operators. Cambridge Tracts in Mathematics, Cambridge University Press, 2009.

[5] H.P. Beise and J. Müller, Generic boundary behaviour of Taylor series in Hardy and Bergman spaces, Math. Z., to appear, arXiv:1512.07397.

[6] L. Bernal-González, Lineability of universal divergence of Fourier series, Integr. Equ. Oper. Theory 74 (2012), 271-279.

[7] L. Bukovsky, The Structure of the Real Line. Birkhäuser, Basel, 2011.

[8] L. Carleson, On convergence and growth of partial sums of Fourier series. Acta Math. 116 (1966), 135-157.

[9] K. Grosse-Erdmann and A. Peris, Linear Chaos. Springer, London, 2011.

[10] G. Herzog and P.C. Kunstmann, Universally divergent Fourier series via Landau's extremal functions. Comment. Math. Univ. Carolinae 56 (2015), 159-168.

[11] K. Hoffman, Banach spaces of analytic functions, Dover, New York, 1962.

[12] J.P. Kahane, Baire's category theorem and trigonometric series. J. Anal. Math. 80 (2000), 143-182.

[13] J.P. Kahane and V. Katznelson, Sur les ensembles de divergence des séries trigonometriques. Studia Math. 26 (1966), 305-306.

[14] E. Katsoprinakis, V. Nestoridis, C. Papachristodoulos, Universality and Cesàro summability. Comput. Methods Funct. Theory 12 (2012), 419-448.

[15] T.W. Körner, Fourier Analysis. Cambridge University Press, 1988.

[16] J. Müller, Continuous functions with universally divergent Fourier series on small subsets of the circle. C.R. Acad. Sci. Paris Ser. I 348 (2010), 1155-1158.

[17] J. Munkres, Topology. 2nd edition, Prentice Hall, Upper Saddle River, NJ, 2000.

[18] J.C. Oxtoby, Measure and Category, 2nd edition, Springer-Verlag, New York, 1980.

[19] Ch. Papachristodoulos, M. Papadimitrakis, On universality and convergence of the Fourier series of functions in the disc algebra, J. Anal. Math., to appear, arXiv:1503.03426v2.

[20] R.W. Vallin, The elements of Cantor sets: with applications, Wiley, Hoboken, 2013.

[21] A. Zygmund, Trigonometric series. Vol. I, II. Cambridge University Press, Cambridge, 2002. 
Luis Bernal-González

Departamento de Análisis Matemático

Facultad de Matemáticas, Apdo. 1160

41080 Sevilla, Spain

e-mail: Ibernal@us.es

Andreas Jung

Fachbereich IV Mathematik

Universität Trier

D-54286 Trier, Germany

e-mail: s4anjung@uni-trier.de

Jürgen Müller

Fachbereich IV Mathematik

Universität Trier

D-54286 Trier, Germany

e-mail: jmueller@uni-trier.de 